\title{
Symmetric/asymmetric bifurcation behaviours of a bogie system
}

\author{
Xue-jun, Gao; Ying-hui, Li; Yuan, Yue; True, Hans
}

Published in:

Journal of Sound and Vibration

Link to article, DOI:

10.1016/j.jsv.2012.09.011

Publication date:

2013

Link back to DTU Orbit

Citation (APA):

Xue-jun, G., Ying-hui, L., Yuan, Y., \& True, H. (2013). Symmetric/asymmetric bifurcation behaviours of a bogie system. Journal of Sound and Vibration, 332(4), 936-951. https://doi.org/10.1016/j.jsv.2012.09.011

\section{General rights}

Copyright and moral rights for the publications made accessible in the public portal are retained by the authors and/or other copyright owners and it is a condition of accessing publications that users recognise and abide by the legal requirements associated with these rights.

- Users may download and print one copy of any publication from the public portal for the purpose of private study or research.

- You may not further distribute the material or use it for any profit-making activity or commercial gain

- You may freely distribute the URL identifying the publication in the public portal

If you believe that this document breaches copyright please contact us providing details, and we will remove access to the work immediately and investigate your claim 


\title{
Symmetric/Asymmetric Bifurcation Behaviours of a Bogie System
}

\author{
Gao Xue-jun ${ }^{1}$, Li Ying-hui ${ }^{2}$, Yue Yuan ${ }^{2}$, Hans True ${ }^{3}$ \\ 1. College of Environment and Civil Engineering, Chengdu University of Technology, Chengdu 610059, China; \\ 2. School of Mechanics and Engineering, Southwest Jiaotong University, Chengdu 610031, China; \\ 3. DTU Informatics, The Technical University of Denmark, DK-2800 Kgs. Lyngby, Denmark
}

\begin{abstract}
Based on the bifurcation and stability theory of dynamical systems, the symmetric/asymmetric bifurcation behaviours and chaotic motions of a railway bogie system under a complex nonlinear wheel-rail contact relation are investigated in detail by the 'resultant bifurcation diagram' method with slowly increasing and decreasing speed. It is found that the stationary equilibrium solution and the periodic motions coexist due to the sub-critical Hopf bifurcation in the railway bogie system. It is also found that multiple solutions coexist in many speed ranges. The coexistence of multiple solutions may result in a jump and hysteresis of the oscillating amplitude for different kinds of disturbances. It should be avoided in the normal operation. Furthermore, it is found that symmetry-breaking of the system through a pitchfork bifurcation leads to asymmetric chaotic motions in the railway bogie system. The speed ranges of asymmetric chaotic motions are, however, small.
\end{abstract}

Key words: symmetry/asymmetry; bifurcation; railway bogie; wheel-rail contact relation; coexistence Author resume: Gao Xue-jun(1979-), male, Ph.D., +86-28-84078955, gaoxj3000@sina.com

\section{Introduction}

With the introduction of CRH (China Railways High-speed) trains, the dynamic performance of the vehicle system becomes more complex. The lateral stability [1-3] of the vehicles is a very important aspect of the vehicle performance. The critical speed of the vehicle, defined as the lowest speed for which self-excited oscillations of the vehicle are possible, is an important design parameter for the vehicle. It directly affects the maximum permissible running speed of the vehicle. The critical speed depends on many factors $[4,5]$, such as the vehicle construction, the track structure, the wheel-rail contact relation, the coefficient of adhesion, the driving torque, and the topology of the railway line. In normal service, passenger cars usually run at speeds lower than the critical speed. It may however happen that the critical speed has decreased below the operating speed due to heavily worn wheel profiles or other reasons, so it is important to know what may happen at speeds higher than the critical speed. 
Much research of bifurcation behaviours and chaotic motions in railway vehicle systems has been carried out at home and abroad. Kaas-Petersen et al. [6] studied the lateral motion of the Cooperrider bogie with and without a flange force. The wheels were assumed to be conical, and the rail profile was assumed to be a circular arc. With a flange force that was modelled as a very stiff spring with a linear characteristic and a dead band, Kaas-Petersen found that chaotic motions exist and that the system changes into an asymmetric periodic motion state through a symmetry-breaking bifurcation. Jensen et al. [7] and Isaksen and True [8] extended the dynamical investigations to unrealistically high speeds in order to follow the development of the dynamics into full chaos through a number of bifurcations and transitions. The development of the quasi-periodic behaviour and the chaos was however strongly influenced by the non-smooth action of the flange force, which created an elastic impact. In contrast to these papers [6-8], this paper uses a model of the wheel-rail contact with realistic wheel and rail profiles. On the basis of the Cooperrider bogie, True and his collaborators [9] established their own research model and investigated its bifurcation behaviour. They found that the sub-critical Hopf bifurcation is very common in the dynamics of four-axle bogie vehicles. True et al. [10] investigated the quasi-stationary dynamics in curves of the Cooperrider bogie with a realistic wheel-rail contact geometry relation. They found that the critical speed in curves can be lower than the permitted speed in the curve and lower than the critical speed on tangent track. Depending on the radius of the curve and the super-elevation of the track, they found a jump between a sub-critical bifurcation/fold bifurcation pair and a single super-critical bifurcation. Yang et al. [11] investigated the Hopf bifurcation and hunting stability of a bogie and locomotive system with hysteretic and nonlinear suspensions. Gao and his supervisors [12] discussed the continuation method and its application in hunting motions and bifurcation behaviours of a six-axle locomotive. Gao et al. [13] also investigated the lateral bifurcation behaviours of a four-axle railway passenger car and found that symmetric/asymmetric periodic and chaotic motions coexist in large speed ranges. They also found that the attractor undergoes symmetry-breaking and symmetry-restoring repeatedly and goes into chaotic motions through period doublings or quasi-periodic motions. The linear and simple nonlinear wheel-rail contact relations were employed to simulate the wheel-rail contact forces in these studies, in which some factors of wheel-rail contact, such as the difference of lengths of the semi axes of the contact ellipse and the change of the normal force were adopted in a crude approximate way, and the dependence of the Kalker creep coefficients on the contact geometry was neglected. In other words, the used wheel-rail contact relation was a relatively crude approximation of the realistic wheel-rail contact state.

The effects of these nonlinear factors were noticed in recent studies, and a research of the dynamics of vehicles with complex nonlinear wheel-rail contact relations was performed by many scientists. Zeng et al. [14] 
studied the linear and nonlinear critical speeds of a dynamical railway vehicle system and found also that the hysteresis phenomenon exists in a realistic vehicle dynamical system. They again found that the system may enter into the chaotic motion through a quasi-periodic motion. Xia's thesis [15] dealt with the dynamics of the Three-Piece-Freight-Truck. The dry friction on the surfaces of the wedges in the suspension system was modelled as two-dimensional dry friction with stick/slip, in contrast to earlier studies where the friction force acted along a line. Hoffmann [16] modelled all kinds of non-smoothness as 'events' and used the 'event system' to formulate the segmented differential equations in different intervals. The integration over the entire domain of definition is thus obtained by a combination of the integrations over the segments. The relations between the segments were defined by the formulas for the non-smoothness. Hoffmann [17] also studied the fundamental dynamic behaviours of European two-axle railway wagons on tangent track. The dynamical investigations of the European two-axle railway wagons were extended to the behaviour in curves by Gialleonardo et al. [18]. They also found - not unexpectedly - that the dynamics of the wagon depends on its position in a rake of wagons, whether it is in the rake or it is at the tail end of the rake. In both cases the dynamics is different from the dynamics of the single wagon.

In fact, the real trouble in railway vehicle dynamics comes from the wheel-rail contact. Hertz presented his contact theory [19] in the late 19th century. According to the Hertz contact theory, the curvatures of the wheel and rail surfaces are constant along the contact patch, and the contact patch can be approximated by an ellipse. It is widely used in railway vehicle dynamics due to its simplicity. However, the result may not be precise if Hertz's assumptions are not fulfilled. One of the biggest limitations is that the contact patch must be elliptic because the shape of the contact patch depends on the local values of the curvature of the two contacting bodies in the contact point [20]. Therefore the semi-Hertizian contact method is applied to overcome this shortcoming. Kik [21] approximates the non-elliptic contact patch by a discrete extension of the Hertz contact theory. Similar models have been developed by Ayasse et al. [22] and Quost et al. [23].

All the theories above can be called non-conformal contact since it is assumed that the contact patch is small compared to the size of the contacting bodies, which both are convex. If one of the bodies is concave, then the conformal contact occurs. Li and Kalker [24] used two quasi-quarter spaces for the numerical solution of the 
conformal contact between wheels and rails.

It is clear that the contact conditions vary considerably depending on the shape of the wheel and rail profiles

[25]. Ideally the contact may take the form of a one-point-contact, a two-point-contact or a multi-point contact.

One-point-contact occurs between the conical or worn tread wheel profiles and the rounded rail profile. If the wheels wear quickly towards the local rail shape, the flange of the wheel set may additionally touch the interior side of the rail, this particular situation is known as two-point-contact. In this case, the rolling contact has two different radii and it causes a large slip between the wheels and the rails and a fast flange wear [26]. At some circumstances, there are multi-point contacts $[27,28]$ between the wheels and the rails. This may be happen when the profiles of the wheels and the rails are heavily worn.

In this paper a more complex nonlinear wheel-rail contact relation is used in our analysis of the symmetric/asymmetric bifurcation behaviours in a railway bogie system. It is a better and more realistic approximation of the actual wheel-rail contact than the one that was used by Kaas-Petersen, Jensen and Isaksen and True in the original Cooperrider bogie contact model. The obtained results are appropriate for applications in vehicle design, experiment and operation. They provide a reference basis for further improvements of the vehicle operation by reducing the wheel-rail wear and designing the running gear of high-speed railway passenger cars.

\section{The Dynamic Model}

\subsection{The Nonlinear Wheel-Rail Contact Relation}

Table 1 Relations between some parameters in the wheel-rail contact and the lateral displacement of the wheel set

\begin{tabular}{|c|c|c|c|}
\hline Parameters in the wheel-rail contact & $\begin{array}{l}\text { Linear wheel-rail contact } \\
\text { relation }\end{array}$ & $\begin{array}{l}\text { Simple nonlinear wheel-rail } \\
\text { contact relation }\end{array}$ & $\begin{array}{c}\text { Complex nonlinear } \\
\text { wheel-rail contact relation } \\
\text { (used in the paper) }\end{array}$ \\
\hline The rolling radius for the left or the right wheel $\left(r_{l}, r_{r}\right)$ & linear & nonlinear & nonlinear \\
\hline The contact angle for the left or the right wheel $\left(\delta_{l}, \delta_{r}\right)$ & linear & nonlinear & nonlinear \\
\hline The roll angel of the wheel set $\left(\phi_{w}\right)$ & linear & nonlinear & nonlinear \\
\hline $\begin{array}{l}\text { The lateral distance between the centre of mass of the } \\
\text { wheel set and the left and the right contact point }\left(a_{l}, a_{r}\right)\end{array}$ & constant & constant & nonlinear \\
\hline The four Kalker creepage coefficients $\left(C_{11}, C_{22}, C_{23}, C_{33}\right)$ & constant & constant & nonlinear \\
\hline $\begin{array}{l}\text { The lengths of the major and minor semi axes of the } \\
\text { contact ellipse }(a, b)\end{array}$ & constant & constant & nonlinear \\
\hline The static normal force & constant & constant & nonlinear \\
\hline
\end{tabular}

Table 1 shows the relations between some parameters in the wheel-rail contact and the lateral displacement of 
the wheel set. The linear wheel-rail contact relation implies that the wheel-rail contact geometry parameters, which mainly include the rolling radius and the contact angle for the left and the right wheels, and the roll angle of the wheel set, are linear functions of the lateral displacements of the wheel set, while other factors in wheel-rail contact are kept constant. The simple nonlinear wheel-rail contact relation implies that the wheel-rail contact geometry parameters are nonlinear and may depend in a non-smooth way of the lateral displacement of the wheel set. The applied and complex nonlinear relations are more accurate descriptions of the properties of the real system. They employ the wheel-rail contact table from RSGEO [29] (RSGEO is a tool by Kik that computes the kinematic relations of the wheel-rail contact) for the determination of the kinematic contact values. We consider the standard S1002 wheel profiles shown in Fig. 1(a) running on the standard UIC60 profiles rails shown in Fig. 1(b) canted at 0.025 towards the track centreline. It is assumed that the rails are fixed rigidly to the ground. The wheels and the rails only touch each other in certain regions of the profiles, see Fig. 2. This makes the wheel-rail contact parameters discontinuous functions of the lateral displacements of the wheel set. The contact table not only includes the commonly used nonlinear wheel-rail contact geometry parameters, but also yields the lateral distance between the mass centre of the wheel set and the left and the right contact point, the four Kalker creepage coefficients, the length of the major and minor semi axes of the contact ellipse and the static normal force - all as functions of the lateral displacement of the wheel set. In railway vehicle dynamics, the normal force is perpendicular to the common tangent plane in the wheel-rail contact points for the left and right wheels. The contact surfaces move due to the relative motion between the wheels and the rails. The static normal force is constant so the wheel set is only influenced dynamically by the contact forces and the forces and the attenuation from the suspension [30].

In order to simplify the contact problem, the contact patch is approximated by an ellipse and only one-point-contact case is considered here. Moreover, it is assumed that the contact problem is two-dimensional, that 
is, the influence of yaw angle of the wheel set on the contact parameters is neglected. The contact forces are formulated in the contact coordinate systems that are located in the corresponding contact points.
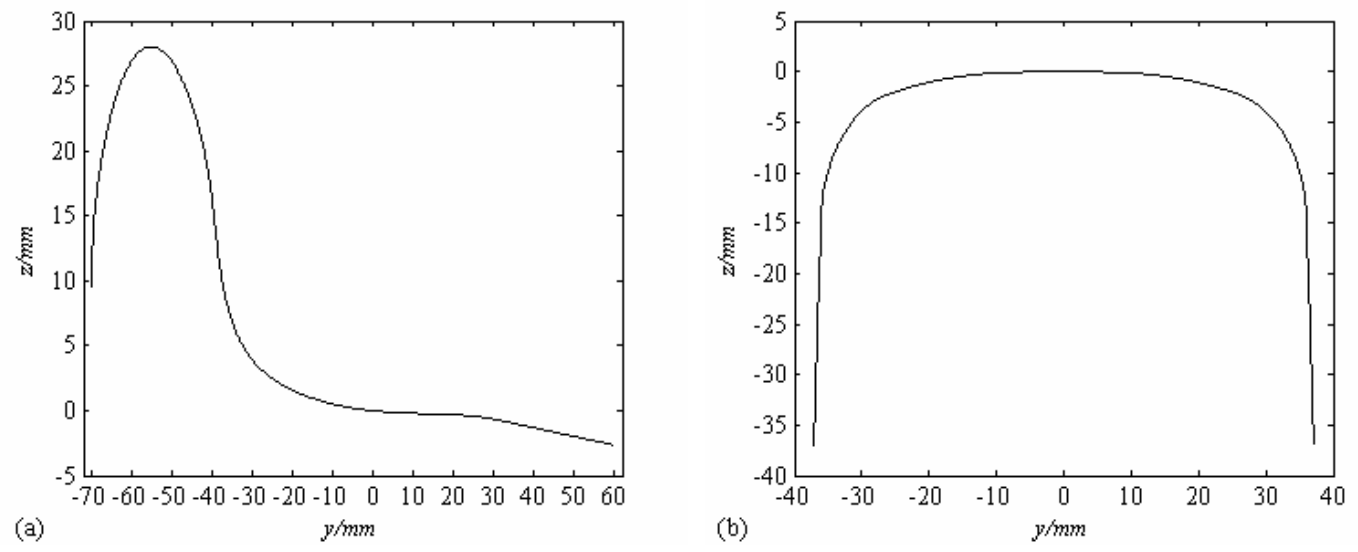

Fig. 1 The standard (a) S1002 wheel profile and (b) the UIC60 rail profile

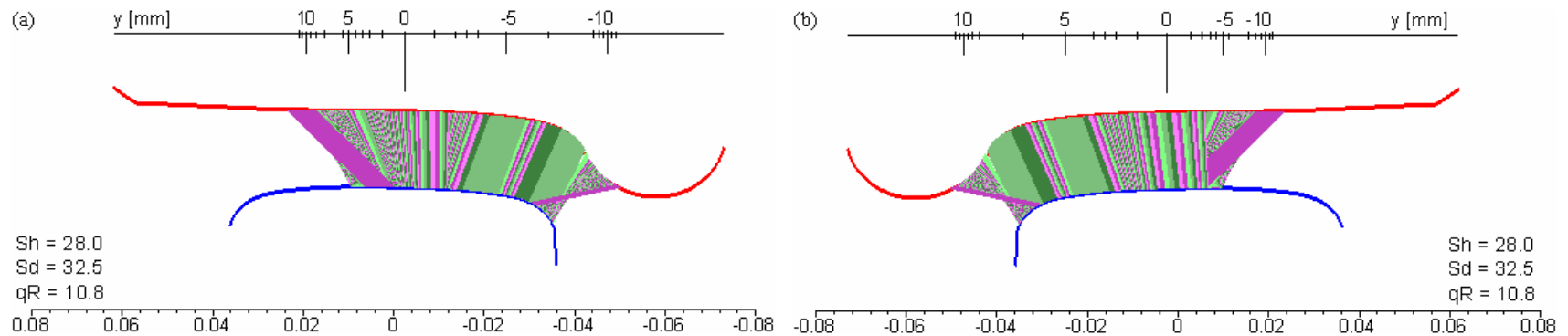

Fig. 2 Possible contact points for (a) the left wheel and rail and for (b) the right wheel and rail. The green areas are the 'no-contact' zones
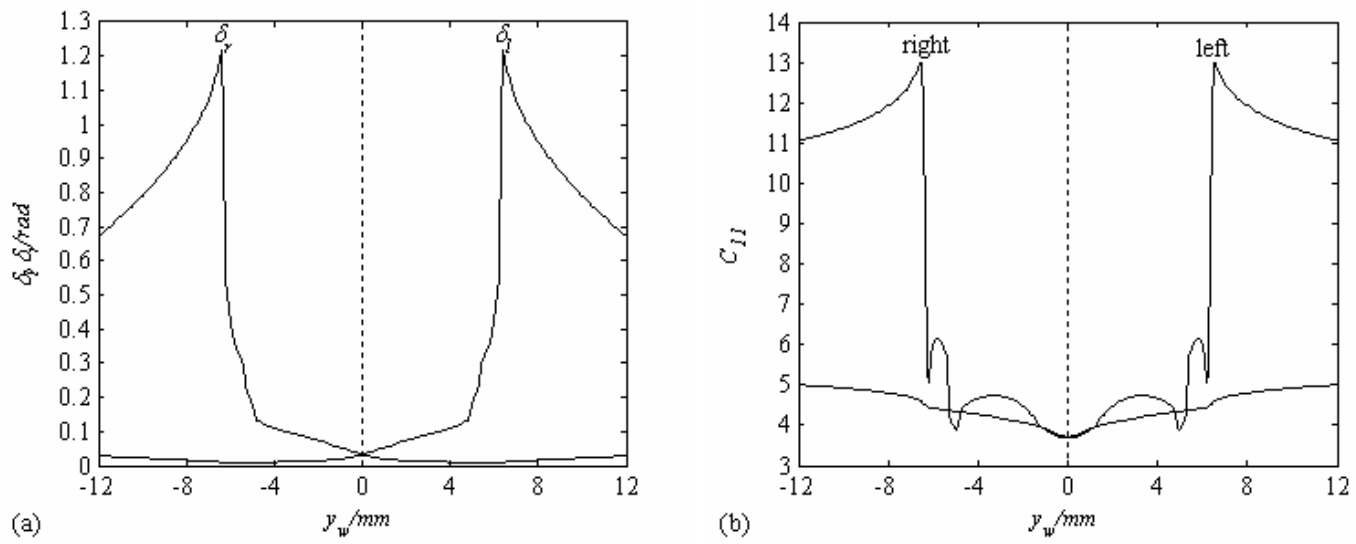

Fig. 3 (a) The contact angle $\left(\delta_{l}, \delta_{r}\right)$ for the left and the right wheel and (b) the Kalker creepage coefficients $C_{11}$ as nonlinear and non-smooth functions of the lateral displacements $y_{w}$ of the wheel set

Fig. 3 shows the contact angle $\left(\delta_{l}, \delta_{r}\right)$ for the left and the right wheel and the Kalker creepage coefficient $C_{11}$ as 
nonlinear functions of the lateral displacements $y_{w}$ of the wheel set. It can be seen from Fig. 3(a) that the contact angle is nearly a piecewise linear function of the lateral displacements of the wheel set when the lateral displacements are small, but it has abrupt changes when the lateral displacements are larger than a certain value, called 'the flange clearance'. Its value is half of the track gauge minus the inside gauge and flange thickness (two sides) in general. We will use the notation $\eta$ for the value of the flange clearance. The contact angles of the left and the right wheel as well as the Kalker creepage coefficients are of course symmetric around the track centreline. The wheel-rail contact parameters are tabulated for the computation of the dynamic behaviour of the railway bogie.

\subsection{The Normal Contact Forces}

The normal contact forces in the contact area deform the wheel and rail to create a contact patch. The contact patch is approximated by an ellipse, which leads to a semi-ellipsoidal normal stress distribution. Two methods are widely used to determine the normal contact forces between the wheels and the rails [27]. One is the rigid model method, which considers the wheels and the rails as rigid bodies, and the other method is the elastic model method, which accounts for the elastic deformation between the wheels and the rails. No doubt the latter method is more realistic.
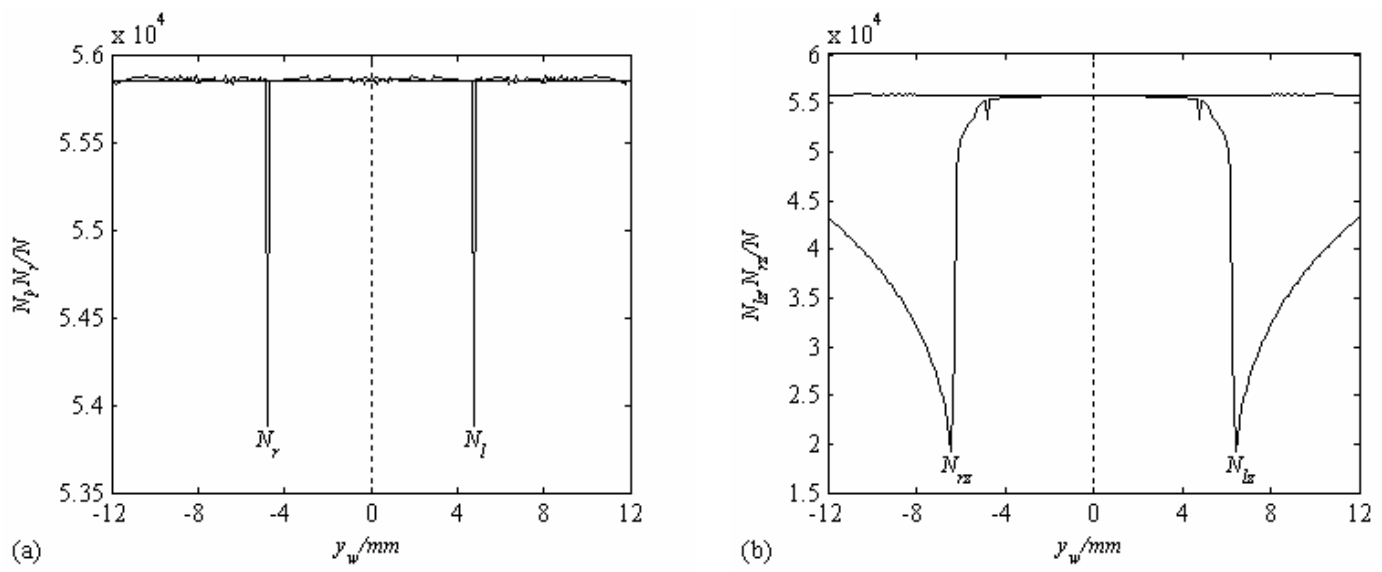

Fig. 4 (a) The static normal forces and (b) their vertical components

In the paper, the vertical degrees of freedom are neglected and the wheels and the rails are assumed to be two 
rigid bodies. The elastic deformation is neglected in order to simplify the dynamic analysis. The static normal contact forces $N_{(l, r)}$ of the wheel sets are directly given in the contact parameter table. They are depicted in Fig. 4(a) as a function of the lateral displacement of the wheel set. It can be seen from the diagram that the static normal forces are almost the same as half of the axle load in most cases, but they change abruptly when the wheel set passes the flange clearance. The lateral components $N_{(l, r) y}$ and the vertical components $N_{(l, r) z}$ of the normal contact forces shown in Fig. 4(b) are then given by

$$
\left\{\begin{array}{c}
N_{(l, r) y}=\mp N_{(l, r)} \sin \left(\delta_{(l, r)} \pm \phi_{w}\right) \\
N_{(l, r) z}=N_{(l, r)} \cos \left(\delta_{(l, r)} \pm \phi_{w}\right)
\end{array}\right.
$$

The subscripts $l$ and $r$ denote the left and right wheel of the wheel set. In the signs \pm and $\mp$ the upper signs apply to the left wheel and the lower signs apply to the right wheel.

The normal contact forces computed in the paper are not very realistic since the wheels and the rails are elastic bodies that are subject to deformation, which causes some mutual penetration in reality.

\subsection{Wheel-Rail Creep Forces and Creep Torques}

A conventional wheel set has two rigid wheels mounted on each end of a rigid axle. The two wheels on the same axle may roll on the two rails with different rolling radii, but they rotate with the same angular speed $\Omega=V / r_{0}$. It is called the nominal angular velocity of the wheel. Here $V$ is the forward speed of the vehicle and $r_{0}$ is the nominal rolling radius. Reference frames are needed to specify the motions of the system. A reference frame moving along the track with the velocity of the bogie is the track coordinate system shown in Fig. 5. The subscript $t$ stands for the track. The coordinate system is a right-hand system, where the directions to the left rail and upwards are positive. The degrees of freedom of the wheel set are smaller than six since the wheel set is constrained (if there is no derailment) to be in contact with the rails. 


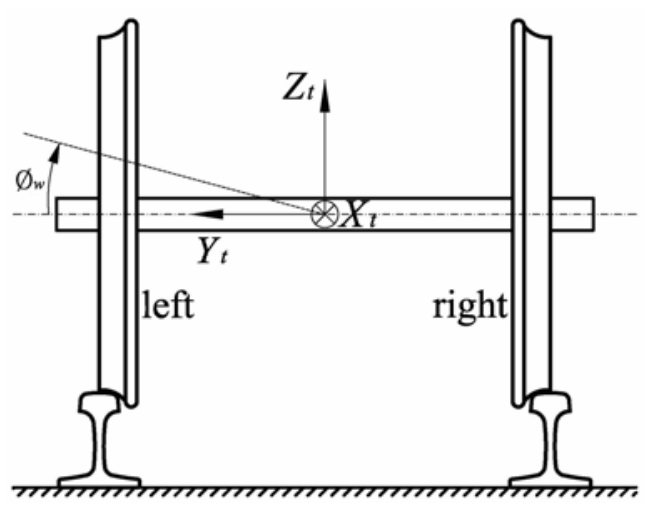

Fig. 5 The track coordinate system

The wheel slides relative to the rail in the contact area between the wheels and the rails, and the relative sliding velocity is called the creep. The creep normalized by the forward speed of the vehicle is denoted the creepage, and the resulting creep force and creep torque both depend on the creepage. The longitudinal creepage $\xi_{x}$, the lateral creepage $\xi_{y}$ and the spin creepage $\xi_{s p}$ of a wheel are [31]

$$
\left.\begin{array}{l}
\xi_{x(l, r)}=\frac{V+\psi_{w} \dot{y}_{w}+r_{(l, r)}\left(\psi_{w} \dot{\phi}_{w}-\Omega\right) \mp a_{(l, r)} \dot{\psi}_{w}}{V} \\
\xi_{y(l, r)}=\frac{-V \psi_{w}+\dot{y}_{w}+r_{(l, r)} \dot{\phi}_{w}}{V \cos \left(\delta_{(l, r)}\right)} \\
\xi_{s p(l, r)}=\frac{\mp\left(\Omega-\psi_{w} \dot{\phi}_{w}\right) \sin \left(\delta_{(l, r)}\right)+\dot{\psi}_{w} \cos \left(\delta_{(l, r)}\right)}{V}
\end{array}\right\}
$$

where in the signs $\mp$ the upper sign apply to the left wheel, and the lower sign to the right wheel. The wheel-rail contact parameters $a_{(l, r)}$ are the lateral distances between the centre of mass of the wheel set and the left or the right contact point. $\psi_{w}$ is the yaw angle of the wheel set.

The nonlinear Shen-Hedrick-Elkins creep model [32], which combines the Kalker linear creep theory with a nonlinear model, is applied in the analysis. According to the Kalker linear creep theory [33], the wheel-rail creep forces in the contact patch are:

$$
\left.\begin{array}{rl}
F_{x} & =-f_{11} \xi_{x} \\
F_{y} & =-f_{22} \xi_{y}-f_{23} \xi_{s p} \\
M_{z} & =f_{23} \xi_{y}-f_{33} \xi_{s p}
\end{array}\right\}
$$


where $F_{x}$ and $F_{y}$ are the longitudinal and lateral creep forces and $M_{z}$ is the spin creep torque. $f_{11}, f_{22}, f_{23}$ and $f_{33}$ are the longitudinal, lateral, lateral/spin and spin creep coefficients that depend on the wheel-rail contact geometry. They are defined as follows:

$$
\left.\begin{array}{l}
f_{11}=G(a b) C_{11} \\
f_{22}=G(a b) C_{22} \\
f_{23}=G(a b)^{3 / 2} C_{23} \\
f_{33}=G(a b)^{2} C_{33}
\end{array}\right\}
$$

Here $G$ is the resultant shear modulus of the wheel and the rail, which are made of the same materials. The symbols $a$ and $b$ are the lengths of the major and minor semi axes of the contact ellipse, and $C_{i j}$ are the Kalker coefficients. These parameters are listed in the contact table from which their interpolated values are taken for the calculations.

The linear creep force components and the spin creep torque in the contact area are found from equation (3) when the creepages are calculated from equation (2). Then the theory by Shen et al. [32] is applied in order to revise the linear results so that the obtained nonlinear creepage/creep force relation can be applied to values of the creepage from zero up to total wheel slip. The revision coefficient $\varepsilon$ is defined as

$$
\varepsilon=\left\{\begin{array}{cc}
(1 / \beta)\left[\beta-\beta^{2} / 3+\beta^{3} / 27\right] & \beta \leq 3 \\
1 / \beta & \beta>3
\end{array}\right.
$$

If $\mu$ is the coefficient of adhesion between wheels and rails, and $N$ is the normal contact forces in the contact patch ( $N_{l}$ for the left wheel and $N_{r}$ for the right wheel as discussed above), then $\beta$ is given by

$$
\beta=\sqrt{F_{x}^{2}+F_{y}^{2}} /(\mu N)
$$

Then the revised creep forces and creep torques are

$$
F_{x}^{\prime}=\varepsilon F_{x}, F_{y}^{\prime}=\varepsilon F_{y}, M_{z}^{\prime}=\varepsilon M_{z}
$$

The transformations between the contact coordinate systems and the track system are applied to obtain the resulting creep forces and creep torques in the track coordinate system. The detailed transformations are [34] 


$$
\left.\begin{array}{l}
F_{(l, r) x}=F_{(l, r) x}^{\prime} \cos \left(\psi_{w}\right)-F_{(l, r) y}^{\prime} \cos \left(\delta_{(l, r)} \pm \phi_{w}\right) \sin \left(\psi_{w}\right) \\
F_{(l, r) y}=F_{(l, r) x}^{\prime} \sin \left(\psi_{w}\right)+F_{(l, r) y}^{\prime} \cos \left(\delta_{(l, r)} \pm \phi_{w}\right) \cos \left(\psi_{w}\right) \\
M_{(l, r) z}=M_{(l, r) z}^{\prime} \cos \left(\delta_{(l, r)} \pm \phi_{w}\right)
\end{array}\right\}
$$

where in the signs \pm the upper sign is for the left wheel and the lower sign for the right wheel.

\subsection{The Model and the Dynamical Equations of the Bogie System}

A two-axle railway bogie system is investigated, and the model is shown in Fig. 6. It consists of a bogie frame with the mass $M_{t}$ and the moments of inertia $I_{t x}, I_{t z}$ and the two wheel sets with the mass $M_{w}$ and the moment of inertia $I_{w x}, I_{w z}$. The primary suspension between the bogie frame and the wheel sets consists of the linearly elastic springs with the characteristics $\left(K_{p x}, K_{p y}, K_{p z}\right)$ and the dampers with the constant characteristics $\left(C_{p x}, C_{p y}, C_{p z}\right)$. They operate in three directions. The secondary suspension also consists of linearly elastic springs with the characteristics $\left(K_{s x}, K_{s y}, K_{s z}\right)$ and the dampers with the constant characteristics $\left(C_{s x}, C_{s y}, C_{s z}\right)$. They also operate in three directions and are mounted between the bogie frame and the car body. (We assume that the car body moves along the track centre line with the constant speed $V$ ). It is assumed that all the parts except the suspension elements are rigid bodies. To simplify the analysis, we make the following assumptions [34]:

(1) The vertical and pitch motions of the bogie frame are neglected, and only the lateral, roll and yaw motions are taken into consideration.

(2) The simulations performed neglect any track irregularities and assume that the wheels roll on a smooth, level, and perfect track.

(3) The vertical displacements are so small that the dynamical equations for the vertical and horizontal motions respectively are uncoupled except for the roll motion of the wheel sets.

(4) Half the length of the primary longitudinal spring arm and damping arm are both equal to $d_{w}$, and half the length of the secondary longitudinal spring arm and damping arm are both equal to $d_{s}$. 
(a) Front view
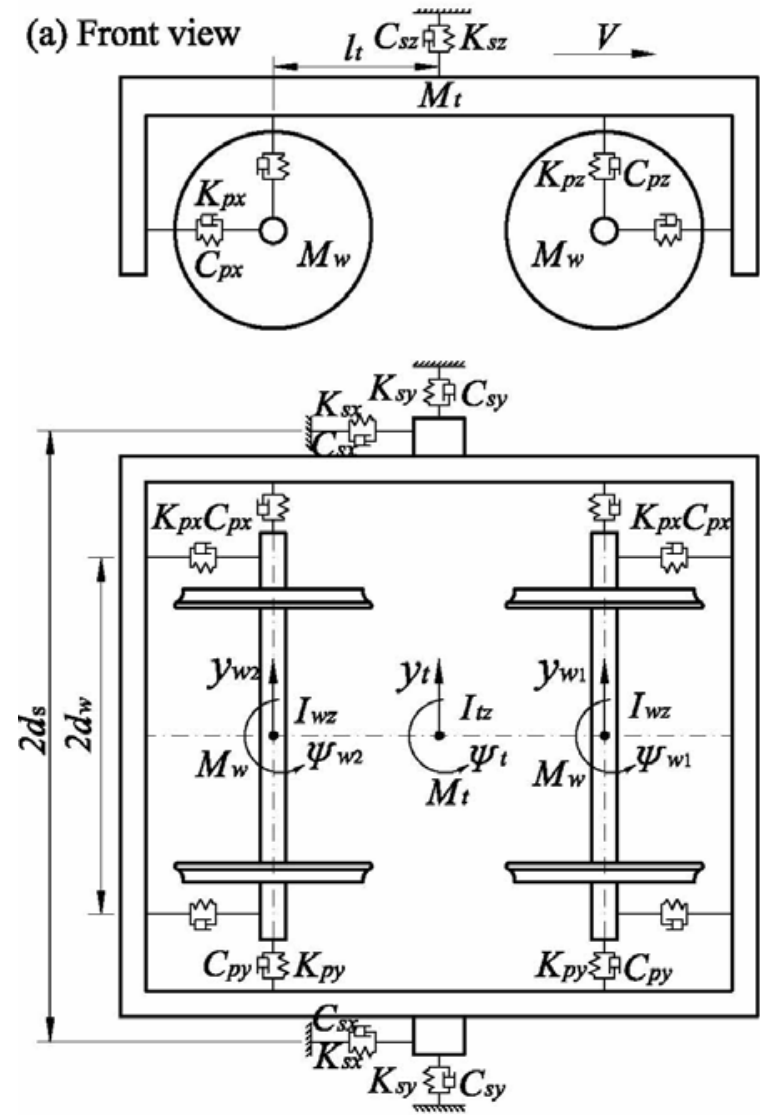

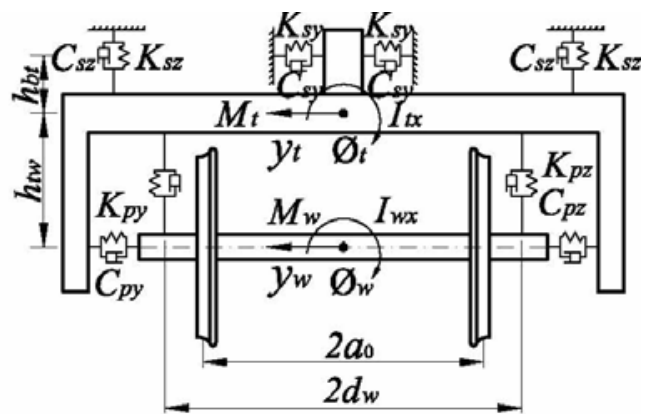

(b) Side view

(c) Top view

Fig. 6 A model of the two-axle railway bogie system

There are seven degrees of freedom in the model as it was the case in the original Cooperrider model by Kaas-Petersen [6]. They are: The lateral motion $y_{t}$, the roll angle $\phi_{t}$ and the yaw angle $\psi_{t}$ of the bogie frame, and the lateral motion $y_{w i}(i=1-2)$ and the yaw angle $\psi_{w i}$ of the two wheel sets. We use Newton's second law to formulate a mathematical dynamical model of the railway bogie system.

Under the assumption that the relative motions between the rigid bodies are small, the following expressions can be obtained. The relative displacements between the wheel sets and the bogie frame are for the lateral motion, the roll angle and the yaw angle respectively

$$
\left.\begin{array}{rl}
U_{i} & =y_{w i}-y_{t} \mp l_{t} \psi_{t}-h_{t w} \phi_{t} \\
V_{i} & =\phi_{w i}-\phi_{t} \\
W_{i} & =\psi_{w i}-\psi_{t}
\end{array}\right\}
$$

where the subscript $i=1,2$ in the physical quantities represents the leading and trailing wheel sets of the bogie. In 
the sign $\mp$ the upper sign is taken if $i=1$, and the lower sign if $i=2$.

The primary suspension forces in the longitudinal, lateral and vertical directions are then given by

$$
\left.\begin{array}{l}
F_{x f i}=2 K_{p x} d_{w} W_{i}+2 C_{p x} d_{w} \dot{W}_{i} \\
F_{y f i}=2 K_{p y} U_{i}+2 C_{p y} \dot{U}_{i} \\
F_{z f i}=2 K_{p z} d_{w} V_{i}+2 C_{p z} d_{w} \dot{V}_{i}
\end{array}\right\}
$$

Similarly the relative lateral displacement, the roll angle and the yaw angle between the bogie frame and the car body are

$$
\left.\begin{array}{rl}
U_{t} & =y_{t}-h_{b t} \phi_{t} \\
V_{t} & =\phi_{t} \\
W_{t} & =\psi_{t}
\end{array}\right\}
$$

The secondary primary suspension forces in the longitudinal, lateral and vertical directions are then given by

$$
\left.\begin{array}{l}
F_{x t}=2 K_{s x} d_{s} W_{t}+2 C_{s x} d_{s} \dot{W}_{t} \\
F_{y t}=2 K_{s y} U_{t}+2 C_{s y} \dot{U}_{t} \\
F_{z t}=2 K_{s z} d_{s} V_{t}+2 C_{s z} d_{s} \dot{V}_{t}
\end{array}\right\}
$$

Then the equations of motion for the railway bogie system can be written in the inertial track coordinate system as

$$
\left.\begin{array}{l}
m_{w} \ddot{y}_{w i}=-F_{y f i}+F_{l y i}+F_{r y i}+N_{l y i}+N_{r y i} \\
I_{w z} \ddot{\psi}_{w i}=-d_{w} F_{x f i}+a_{0}\left(F_{r x i}-F_{l x i}\right)+a_{0} \psi_{w i}\left(F_{r y i}\right. \\
\left.\quad+N_{r y i}-F_{l y i}-N_{l y i}\right)+M_{l z i}+M_{r z i}-I_{w y} \dot{\phi}_{w i} \Omega \\
m_{t} \ddot{y}_{t}=F_{y f 1}+F_{y f 2}-F_{y t} \\
I_{t z} \ddot{\psi}_{t}=l_{t}\left[F_{y f 1}-F_{y f 2}\right]+d_{w}\left[F_{x f 1}+F_{x f 2}\right]-d_{s} F_{x t} \\
I_{t x} \ddot{\phi}_{t}=h_{t w}\left[F_{y f 1}+F_{y f 2}\right]+d_{w}\left[F_{z f 1}+F_{z f 2}\right] \\
\quad+h_{b t} F_{y t}-d_{s} F_{z t}
\end{array}\right\}
$$

where $F_{(l, r) x}$ and $F_{(l, r) y}$ are the longitudinal and lateral creep forces and $M_{(l, r) z}$ is the spin creep torque.

The displacement, velocity and acceleration vectors of the bogie system are denoted by $\mathbf{x}, \dot{\mathbf{x}}$ and $\ddot{\mathbf{x}}$. They can be defined by 


$$
\left.\begin{array}{l}
\mathbf{x}^{\mathrm{T}}=\left\{y_{w 1}, y_{w 2}, \psi_{w 1}, \psi_{w 2}, y_{t}, \psi_{t}, \phi_{t}\right\} \\
\dot{\mathbf{x}}^{\mathrm{T}}=\left\{\dot{y}_{w 1}, \dot{y}_{w 2}, \dot{\psi}_{w 1}, \dot{\psi}_{w 2}, \dot{y}_{t}, \dot{\psi}_{t}, \dot{\phi}_{t}\right\} \\
\ddot{\mathbf{x}}^{\mathrm{T}}=\left\{\ddot{y}_{w 1}, \ddot{y}_{w 2}, \ddot{\psi}_{w 1}, \ddot{\psi}_{w 2}, \ddot{y}_{t}, \ddot{\psi}_{t}, \ddot{\phi}_{t}\right\}
\end{array}\right\}
$$

The equations of motion for the railway bogie system then yield seven coupled nonlinear second order ordinary differential equations (ODEs)

$$
\mathbf{M} \ddot{\mathbf{x}}+\mathbf{C} \dot{\mathbf{x}}+\mathbf{K x}=\mathbf{P}(\mathbf{x}, \dot{\mathbf{x}})
$$

where $\mathbf{M}, \mathbf{C}, \mathbf{K}$, and $\mathbf{P}$ are the mass matrix, the damping matrix, the stiffness matrix and the load vectors of the system.

When the state vector $\mathbf{y}^{\mathrm{T}}=\left\{\dot{\mathbf{x}}^{\mathrm{T}}, \mathbf{x}^{\mathrm{T}}\right\}$ is substituted into equation (15), then the dynamical problem of the bogie is formulated as an initial value problem for an autonomous system of fourteen ODEs of first order with the time $t(t>0)$ as the single independent variable.

$$
\frac{\mathrm{d} \mathbf{y}}{\mathrm{d} t}=\mathbf{f}(\mathbf{y}, V)
$$

where $V \in \mathbf{R}^{+}$is the forward speed of the bogie and the control parameter. $\mathbf{f}$ is a function of the state vector.

Given suitable initial conditions $\mathbf{y}_{0}$, we can integrate the system of ODEs (16), and the general solution vector is of the form

$$
\mathbf{y}=\mathbf{y}\left(t, V, \mathbf{y}_{0}\right)
$$

Depending on the speed, we may find stationary and/or periodic and aperiodic solutions that may be stable or unstable. The stationary motion, which is the trivial solution of the dynamical system, is unique and asymptotically stable at a sufficiently low speed. When the speed is increased to a certain value, $V_{H}$, the stable stationary solution loses its stability and a bifurcation of a periodic solution is found. It is sub-critical wherefore a speed interval exists below $V_{H}$, where the stationary solution exists together with two periodic solutions of which one is stable and the other unstable. If the bifurcation had been super-critical, then a stable periodic solution exists for $V>V_{H}$. For still higher speeds multi-periodic and aperiodic motions may appear. It was illustrated and discussed in detail by Gao et 
al. [35] and True [36] etc.

\section{Method of Investigation}

The dynamical system is a nonlinear and non-smooth system due to the nonlinear and non-smooth wheel-rail contact relation and the nonlinear strain velocity-stress relation. Therefore, and because of the number of degrees of freedom, the system must be investigated numerically. The forward speed $V$ of the bogie is chosen as the control parameter in the analysis.

For the investigations of periodic and aperiodic solutions we use a Poincaré section $\prod_{1}$ and its symmetric section $\Pi_{2}$ with slowly increasing or decreasing speed to construct the bifurcation diagram. In this way, however, we can only find the asymptotically stable solution branches. The Poincaré section is defined by

$$
\Pi_{1}=\left\{(\mathbf{y}, V) \in \mathbf{R}^{14} \times \mathbf{R}^{+} \mid \dot{y}_{t}=0, y_{t} \geq 0\right\}
$$

There exists a Poincaré section $\Pi_{2}$ that is a reflection of the original Poincaré section $\Pi_{1}$ at the track centreline. The symmetric Poincaré section is given by

$$
\Pi_{2}=\left\{(\mathbf{y}, V) \in \mathbf{R}^{14} \times \mathbf{R}^{+} \mid \dot{y}_{t}=0, y_{t} \leq 0\right\}
$$

Using the Poincaré sections defined above, we can construct bifurcation diagrams of the lateral amplitudes of the bogie frame and determine whether a solution is periodic or aperiodic, but we cannot determine its symmetry properties. However, asymmetry is also very important in vehicle system dynamics, because it may cause lopsided wear and lead to chaotic motion at higher speeds. Since the entire system has a reflection symmetry at the track centreline, we can reflect the negative lateral amplitude of the bogie frame at the track centreline onto the positive direction and construct the 'resultant bifurcation diagram'. If a solution is symmetric, the branches from the negative and positive $y$-values are identical in the 'resultant bifurcation diagram'. In this way, we can distinguish between symmetric and asymmetric solutions.

For the numerical integration we use a cubic spline interpolation in the contact table to determine the values of 
the nonlinear wheel-rail contact parameters at arbitrary lateral displacements of the wheel set.

For the equation solver, we use a quasi-constant step size implementation [37] (the standard ode15s routine in MATLAB) in terms of backward differences of the Klopfenstein-Shampine family of numerical differentiation formulas of variable order. It uses a variable time step and error control. Interpolation and local extrapolation is done in some cases. In order to interpolate the trajectory between integration steps, an interpolation with the same asymptotic error as the global error of the numerical integration is used. In the paper, the solutions are determined with the absolute and relative errors of order $10^{-7}$.

\section{Numerical Results and Discussion}

In this section, we describe the dynamics of a bogie of a high-speed railway passenger car for the speed range $0<V<90.0 \mathrm{~m} / \mathrm{s}$. The values of the masses of the rigid bodies, the stiffness coefficients, the damping coefficients and the other parameters can be found in Appendix A. The other wheel-rail contact parameters that are relevant for the lateral displacements of the wheel set in the computation are already described in section 2.1. One uncontrollable parameter that is relevant for the wheel-rail creep forces is the coefficient of adhesion. It has a great influence on the vehicle stability and is usually determined from an empirical formula based on a statistical method using experimental data. In the paper we set the value of the coefficient of adhesion to $\mu=0.15$ as it was done in the papers by Kaas-Petersen [6], Jensen [7] and Isaksen [8].

\subsection{Stationary and Symmetric Periodic Motion}

Some results of the bifurcation analysis of the bogie frame in the speed range $65.0 \mathrm{~m} / \mathrm{s}<V<90.0 \mathrm{~m} / \mathrm{s}$ are illustrated in Fig. 7. The dynamic long-time behaviour of the bogie frame versus the forward speed is represented in the bifurcation diagram by the amplitudes of the bogie frame displacements. The stationary solution is the trivial solution. The 'resultant bifurcation diagram' method is used here to construct the bifurcation diagram for a convenient evaluation of the symmetry of motions about the track centreline. The diagram, Fig. 7(a) is calculated for increasing speed, while Fig. 7(b) is calculated for decreasing speed. In these two diagrams, the red points are 
points in the Poincaré section $\prod_{1}$, the black ones are the points reflected in the track centreline from the points in the Poincaré section $\Pi_{2}$. Except for the speed range $83.453 \mathrm{~m} / \mathrm{s}<V<85.361 \mathrm{~m} / \mathrm{s}$ (point $B$ to $C$ ), where some points in the bifurcation diagram Fig. 7(a) in different colours indicate asymmetric motions, the oscillations are symmetrical about the track centreline. The inherent and related nonlinear dynamic behaviours in Fig. 7 are described below.
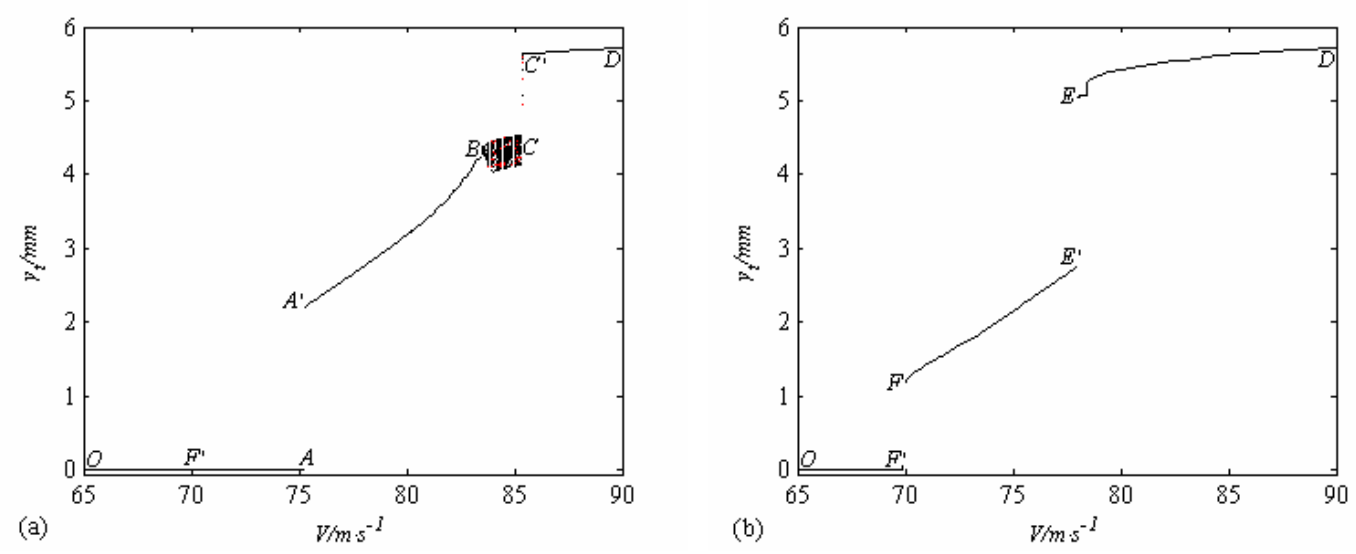

Fig. 7 The 'resultant bifurcation diagram' for the bogie frame with (a) increasing speed and (b) decreasing speed showing the lateral amplitudes versus the speed. Only the asymptotically stable solutions are shown

On Fig. 7(a) the forward speed starts from $V=65.0 \mathrm{~m} / \mathrm{s}$ and increases with small steps of $0.1 \mathrm{~m} / \mathrm{s}$. The initial values of the numerical integration are always chosen to be close to the solution branch we want to follow. It can be seen from the diagram that the system will eventually stabilize at the trivial solution when the speed is small enough. It is an asymptotically stable solution (the stationary solution branch $O A$ in the diagram). On the section $O F^{\prime}$ the trivial solution is also globally unique. When the speed gradually increases and reaches point $A\left(V_{A}=75.3\right.$ $\mathrm{m} / \mathrm{s}$ ), then the trivial solution loses its stability, and the amplitude of the periodic motion of the bogie frame jumps from the point $A$ to $A^{\prime}\left(\max \left|y_{t A}\right|=2.2184 \mathrm{~mm}\right.$ is the lateral amplitude of the periodic motion in the point $\left.A^{\prime}\right)$, and the periodic motion is asymptotically stable. The point $A$ is a sub-critical bifurcation point of the system, from where an unstable periodic solution bifurcates and the stationary solution loses its stability. Due to the differential-algebraic 
nature of the dynamical problem, since the railway bogie is a multi-body system with algebraic constrains, it is not possible to follow that unstable periodic solution in the parameter-state space by any numerical integration method. The amplitude of the stable periodic solution of the bogie frame grows rapidly with increasing speed, until an aperiodic - seemingly chaotic motion develops and the periodic solution disappears at $V_{B}=83.453 \mathrm{~m} / \mathrm{s}$ with $\max \left|y_{t B}\right|=4.241 \mathrm{~mm}$. After that, the bandwidth of the attractor slowly increases with increasing speed. In fact, when the forward speed increases through the speed $V_{B}=83.453 \mathrm{~m} / \mathrm{s}$, the symmetric periodic attractor disappears and the motion jumps to two asymmetric chaotic attractors, which are mirror images of each other. Then follow a lot of different bifurcations to symmetric or asymmetric chaotic motions, such as torus breakdown, exterior and, interior crisis, attractor merging crisis, etc. At last, when the speed is larger than $V_{C}=85.361 \mathrm{~m} / \mathrm{s}$, the chaotic attractor jumps at point $C$ to a symmetric period 1 attractor at $C^{\prime}\left(\max \left|y_{t C}\right|=5.629 \mathrm{~mm}\right)$ and the aperiodic attractor disappears. The detailed analysis of the aperiodic attractor will be discussed in another paper because the 'chaotic part' of the motion is very small compared with the average amplitudes. When the speed is larger than $V_{C}$, the amplitude of the periodic motion of the bogie frame increases with increasing speed until the forward speed of $V_{D}=90.0 \mathrm{~m} / \mathrm{s}$ $\left(\max \left|y_{t D}\right|=5.706 \mathrm{~mm}\right)$ is reached.

Fig. 8 shows the phase trajectories for the periodic motion at $V=83.4 \mathrm{~m} / \mathrm{s}$ and $V=85.4 \mathrm{~m} / \mathrm{s}$. The ordinate is the lateral displacements of the leading wheel set of the bogie and the abscissa is the lateral displacements of the bogie frame. It is seen from Fig. 8(a) that the leading wheel set of the bogie flips back and forth between close flange contacts. On Fig. 8(b), we see that the wheel set alternates between running on the flanges of the right and left wheels. The flange contact is pronounced and creates a small extra bounce of the wheel set. The flange running increases the abrasion and wear of the wheels and the rails and leads to higher maintenance costs. 

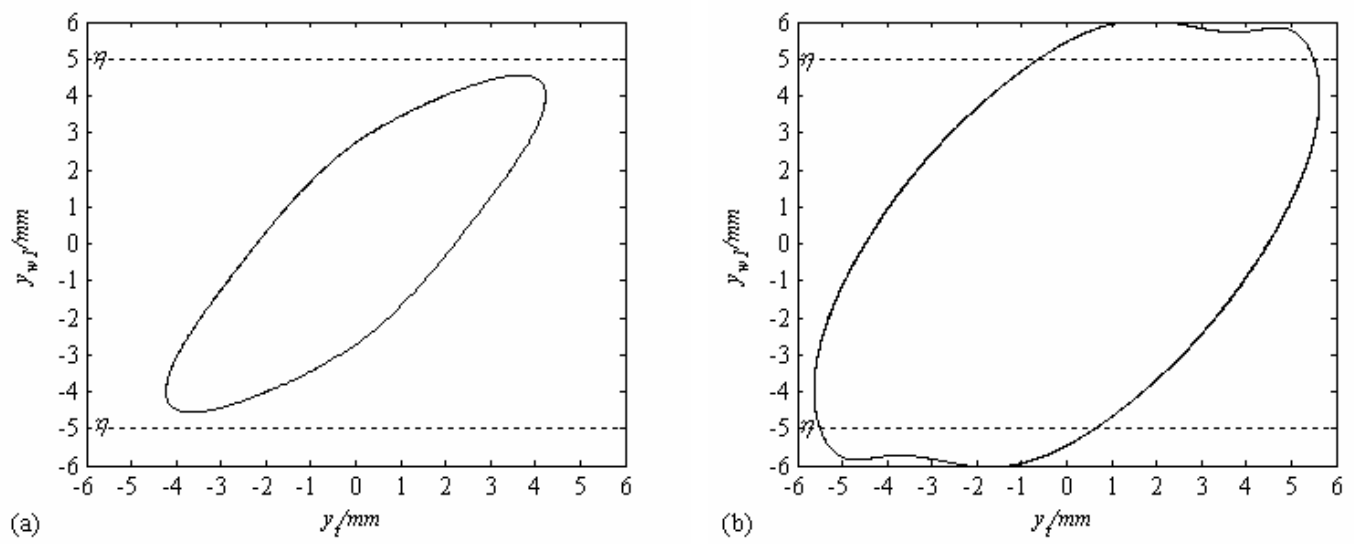

Fig. 8 The phase trajectories for the periodic motion at (a) $V=83.4 \mathrm{~m} / \mathrm{s}$ and (b) $V=85.4 \mathrm{~m} / \mathrm{s}$

The time-history of the wheel-rail contact forces is a very important quantity for the determination of the safety of railway vehicles. The ratio between the guiding force and the wheel load, called Y/Q, is used for an estimate of the danger of derailment. Fig. 9 shows the time series of the wheel load and the guiding force of the leading wheel set of the bogie at $V=85.4 \mathrm{~m} / \mathrm{s}$. It can be seen from Fig. 9(a) that the wheel load decreases from 56.20 $k N$ to $51.81 k N$ in a short time. Please remember that the vertical motion of the bogie frame is ignored in our dynamical system, so the calculated unloading may be smaller than the one of the real bogie. Fig. 9(b) shows the time evolution of the guiding force. The maximum value of the guiding force is $21.32 \mathrm{kN}$. With the minimum value of the calculated wheel load it yields a maximum value of $\mathrm{Y} / \mathrm{Q}=0.41$.

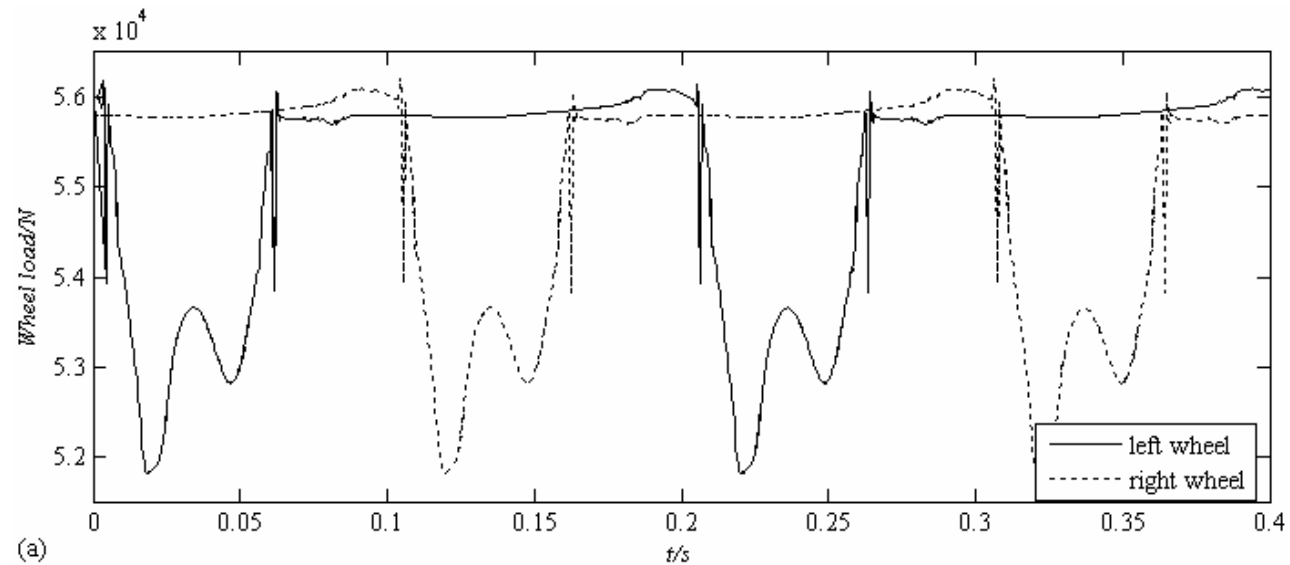




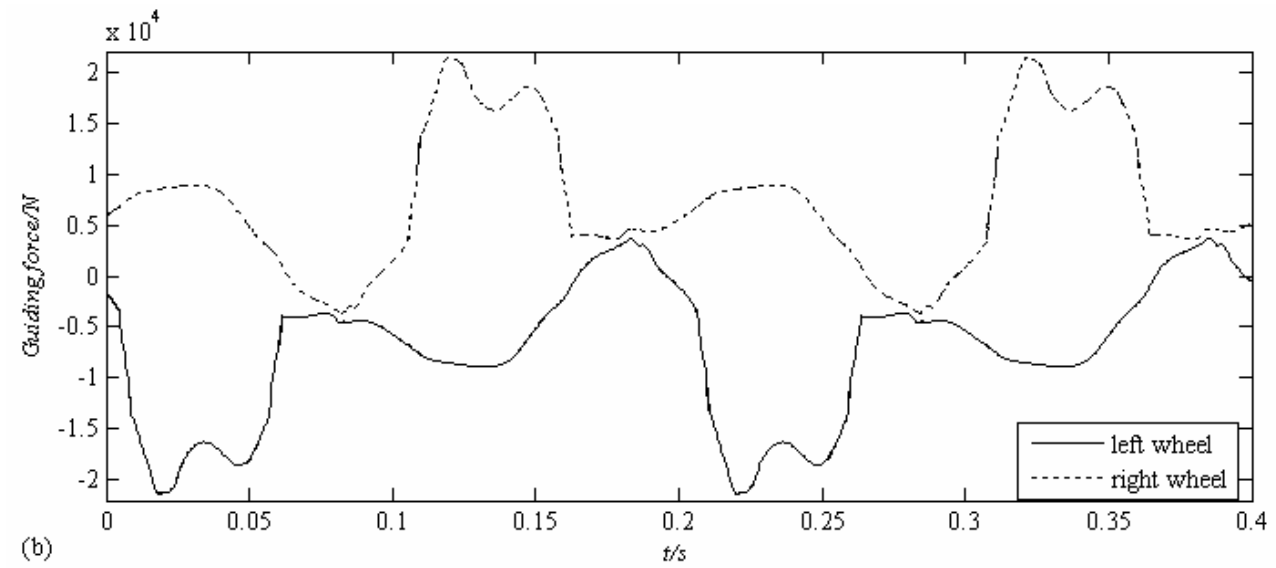

Fig. 9 The time series of (a) the wheel load and (b) the guiding force of the leading wheel set of the bogie at $V=85.4$

$$
m / s
$$

Now we look at the bifurcation diagram Fig. 7(b). Here the speed decreases with small steps of $0.1 \mathrm{~m} / \mathrm{s}$ from $V=90.0 \mathrm{~m} / \mathrm{s}$. The initial values of the numerical integration for the next step are the end values of the state of the previously computed motion after the transient is negligible. The branch will follow the $D E E{ }^{\prime} F F^{\prime} O$ path in Fig. 7(b), and the amplitude of the periodic motion of the bogie frame decreases with decreasing speed until $V_{E}$ $\left(V_{E}=78.0 \mathrm{~m} / \mathrm{s}, \max \left|y_{t E}\right|=5.0585 \mathrm{~mm}\right)$ is reached. After that, a leap from the periodic attractor $E$ to the other periodic attractor $E^{\prime}\left(\max \left|y_{t E}\right|=2.7412 \mathrm{~mm}\right)$ takes place through a fold bifurcation at point $E$. Then the amplitude of the bogie frame decreases rapidly with the continuously decreasing speed until the speed reaches $V_{F}\left(V_{F}=70.0 \mathrm{~m} / \mathrm{s}\right.$, $\left.\max \left|y_{t F}\right|=1.1902 \mathrm{~mm}\right)$, where another leap from the periodic attractor $F$ to the stationary attractor $F^{\prime}\left(V_{F},=69.9 \mathrm{~m} / \mathrm{s}\right)$ takes place through another fold bifucation.

To illustrate the leap from the point $F$ to $F^{\prime}$ in Fig. 7(b), Fig. 10 shows time series of the lateral displacements of the leading wheel set of the railway bogie at $V=70.0 \mathrm{~m} / \mathrm{s}$ (black color) and $V=69.9 \mathrm{~m} / \mathrm{s}$ (red color) with the same initial condition. It can be seen that the leading wheel set of the bogie oscillates periodically with the amplitude $1.1902 \mathrm{~mm}$ as a function of time at $V=70.0 \mathrm{~m} / \mathrm{s}$, while the lateral displacements of the wheel set decays to zero at $V=69.9 \mathrm{~m} / \mathrm{s}$. 


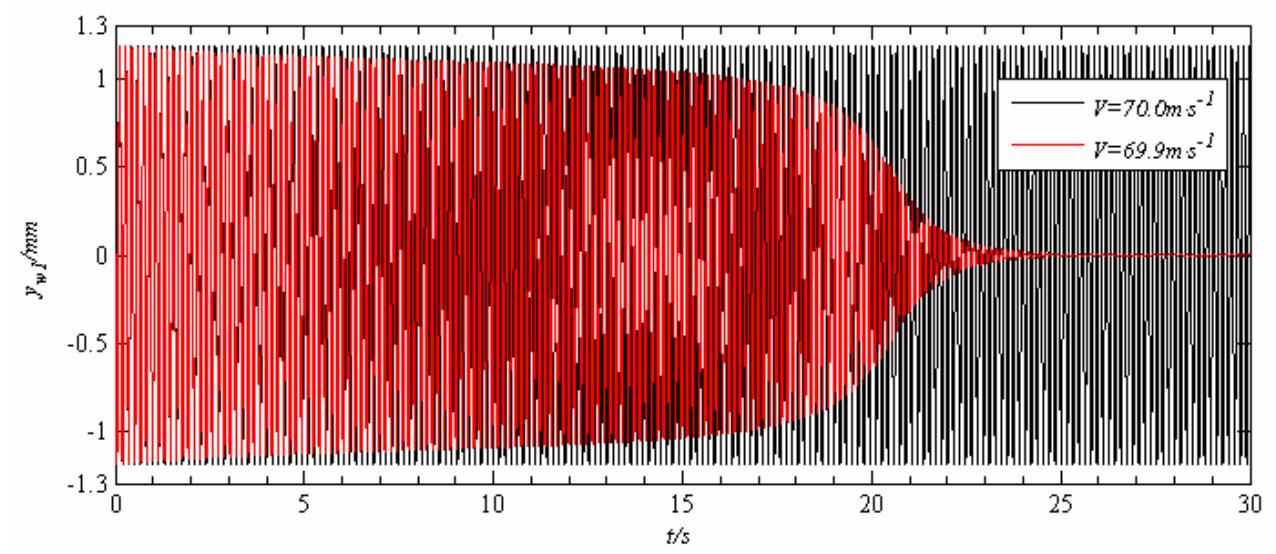

Fig. 10 The time series of the leading wheel set of the railway bogie at $V=70.0 \mathrm{~m} / \mathrm{s}$ and $V=69.9 \mathrm{~m} / \mathrm{s}$

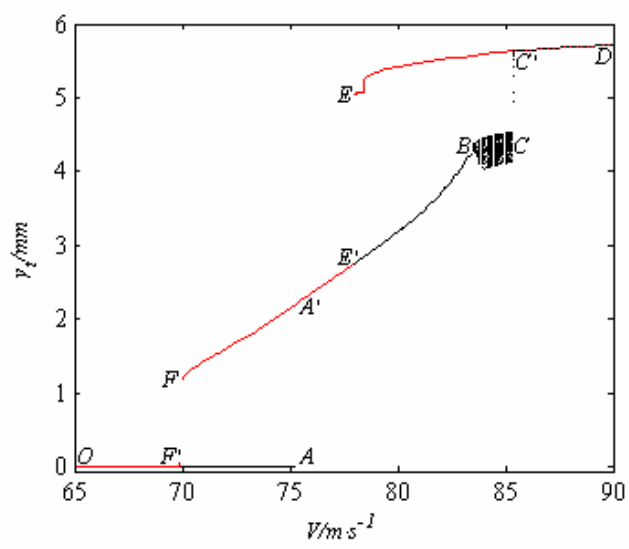

Fig. 11 The complete bifurcation diagram with slowly increasing and decreasing speeds (the black curves for increasing speed and the red ones for decreasing speed)

In order to show the jump and hysteresis of the amplitudes that appear in the system when the speed grows or decays, the complete bifurcation diagram with slowly increasing and decreasing speed is illustrated in Fig. 11. It is plotted from the Poincaré section $\prod_{1}$. The figure is a superposition of the Fig. 7(a) and Fig. 7(b). The diagram is shown in different colours. The black curves are for increasing speed and the red ones are for decreasing speed. It can be seen from the diagram that there are jumps connected with hysteresis in the railway bogie dynamics at some speeds. A hysteresis exists between $F F^{\prime}$ and $A A^{\prime}$ and another between $E E^{\prime}$ and $C C^{\prime}$. We therefore have multiple attractors in the speed ranges $V_{F}<V<V_{A}$ and $V_{E}<V<V_{C}$. In these speed intervals the equilibrium solution of the initial value problem for our dynamical system depends on the initial conditions. In praxis the external disturbances may make the system jump between the steady solutions. In railway vehicle dynamics the oscillation worsens the passenger comfort, and they may in rare cases lead to some potential dangers for the safe operation. Therefore the non-stationary motions should be avoided in the normal operation of the railway vehicles. 
Between the points $A$ and $F$ on Fig. 11 an unstable periodic solution exists. Its amplitude grows from zero in $A$ to a certain value in $F$, where it joins the stable periodic branch in a fold bifurcation. Another unstable periodic solution may exist between the points $C$ and $E$. The unstable branches $A F$ and $C E$ cannot be determined by the numerical integration method as mentioned before, and they are therefore not shown on Fig. 11. The speed in point $A$, at which the stationary solution loses its stability in a Hopf bifurcation, is often called 'the Hopf bifurcation speed'. The value of the speed in point $F$ is the lowest speed for which a periodic motion exists and below which the stationary solution is unique and asymptotically stable. It is the critical speed of the vehicle. It should be used as the highest permitted speed in normal operation for the design of the vehicle.

\subsection{The Role of Symmetry Breaking}

In the bifurcation diagram Fig. 7(a) the 'resultant bifurcation diagram' method with slowly increasing speed is used to plot the bifurcation diagram to show the symmetric/asymmetric bifurcation behaviour and the chaotic motion of the bogie system. The results show that there are symmetric motions as well as a small domain of asymmetric motions in spite of the right/left as well as the front/back symmetry of the bogie model. The front/back symmetry is already broken during the motion however, when the speed is different from zero. The effects of these broken symmetries were investigated and discussed by Jensen et al [38]. The 'resultant bifurcation diagram' method with slowly decreasing speed is now applied to construct the bifurcation diagram in order to show the possible symmetric/asymmetric motions and hysteresis.

The bifurcation diagram in Fig. 12 shows the lateral amplitude of the bogie frame versus the forward speed in the speed interval $83.0 \mathrm{~m} / \mathrm{s}<V<83.5 \mathrm{~m} / \mathrm{s}$. The periodic attractor $E^{\prime} B$ (see Fig. 11) ends in Fig. 12 in a fold bifurcation at $V_{B}=83.453 \mathrm{~m} / \mathrm{s}$. From $B$ an unstable periodic branch bifurcates towards lower speeds and ends in another fold bifurcation at $J\left(V_{J}=83.056 \mathrm{~m} / \mathrm{s}\right)$, where it gains stability. Between $V_{J}=83.056 \mathrm{~m} / \mathrm{s}$ and $V_{I}=83.32 \mathrm{~m} / \mathrm{s}$ there is a hysteresis loop between two periodic attractors with different amplitudes. Between $V_{I}=83.32 \mathrm{~m} / \mathrm{s}$ and $V_{B}=83.453$ $\mathrm{m} / \mathrm{s}$ the loop changes character, because the upper branch splits up into two asymmetric attractors at $V_{I}=83.32 \mathrm{~m} / \mathrm{s}$ and at $V_{H}=83.416 \mathrm{~m} / \mathrm{s}$ a periodic solution doubling sequence for both asymmetric attractors starts. It leads to two asymmetric chaotic attractors. Fig. 13 shows an enlarged portion of Fig. 12 in the speed interval $83.3 \mathrm{~m} / \mathrm{s}<V<83.5$ $\mathrm{m} / \mathrm{s}$. The plot shows the development of the chaotic motion in detail. The amplitude of the two asymmetric attractors grows with increasing speed until the two attractors touch the unstable period 1 solution between them and merge into a broader symmetric chaotic attractor at $V=83.475 \mathrm{~m} / \mathrm{s}$ in an attractor merging crisis [39]. The symmetry breaking bifurcation at $V_{I}=83.32 \mathrm{~m} / \mathrm{s}$ is generic for a symmetric system before chaos can develop through 
local bifurcations as shown by Jensen et al. [38].

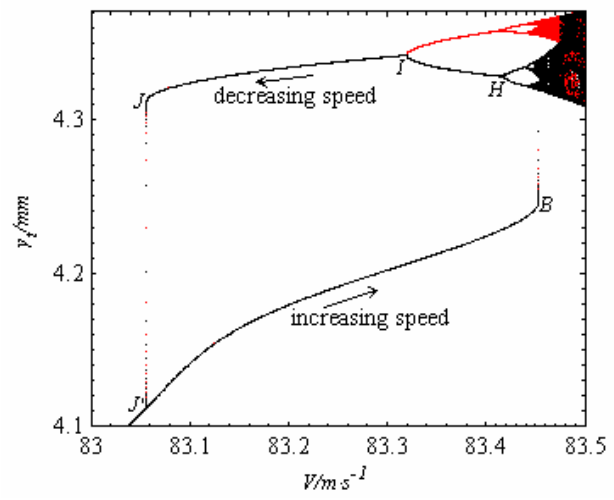

Fig. 12 The bifurcation diagram of the bogie frame in the speed interval $83.0 \mathrm{~m} / \mathrm{s}<V<83.5 \mathrm{~m} / \mathrm{s}$

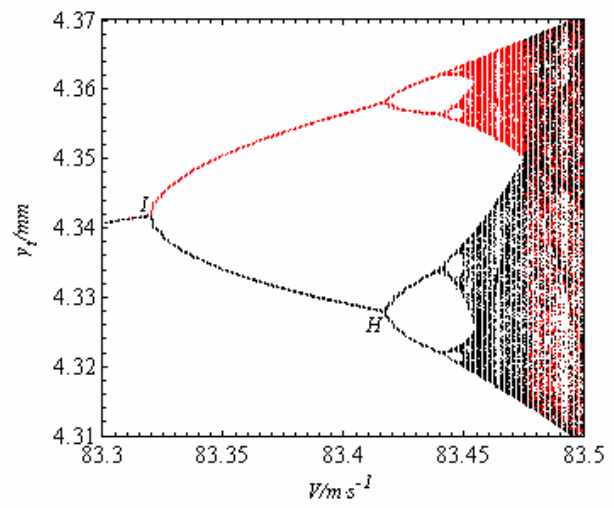

Fig. 13 An enlarged portion of Fig. 12 in the speed interval $83.3 \mathrm{~m} / \mathrm{s}<V<83.5 \mathrm{~m} / \mathrm{s}$

It can be seen from the diagram Fig. 12 that the amplitude of the symmetric periodic attractor decreases with the decreasing speed until the speed reaches $V_{J}=83.056 \mathrm{~m} / \mathrm{s}$ where a jump from the point $J$ to $J^{\prime}$ appears (the point $J^{\prime}$ is a point of solution branch $E^{\prime} B$ in the diagram Fig. 11). If the speed continues to decrease, the symmetric periodic solution branches will follow the $E^{\prime} F F^{\prime} O$ path in Fig. 11. If the speed begins to increase from the speed of point $J^{\prime}$, the symmetric periodic solution will trace the route $J^{\prime} B$ and suddenly jump from a symmetric periodic motion to an asymmetric chaotic motion at $V_{B}=83.453 \mathrm{~m} / \mathrm{s}$ as stated in the section 4.1 .

Fig. 14 shows the time series of the bogie frame at $V=83.46 \mathrm{~m} / \mathrm{s}$ and $V=83.47 \mathrm{~m} / \mathrm{s}$. The two speeds are in the speed interval of $83.453 \mathrm{~m} / \mathrm{s}<V<83.475 \mathrm{~m} / \mathrm{s}$ where there exist two asymmetric chaotic solutions. However, it can hardly be seen on the figures since they both look more like periodic solutions, but they are indeed periodic solutions superposed with a chaotic contribution of very small amplitude. Fig. 15 shows the time series of the bogie frame at $V=83.48 \mathrm{~m} / \mathrm{s}$. It is a symmetric chaotic attractor with a dominating periodic component. The chaotic attractor is therefore hardly more undesirable than the periodic hunting and if so then mainly because of the growing amplitude of the oscillation. 

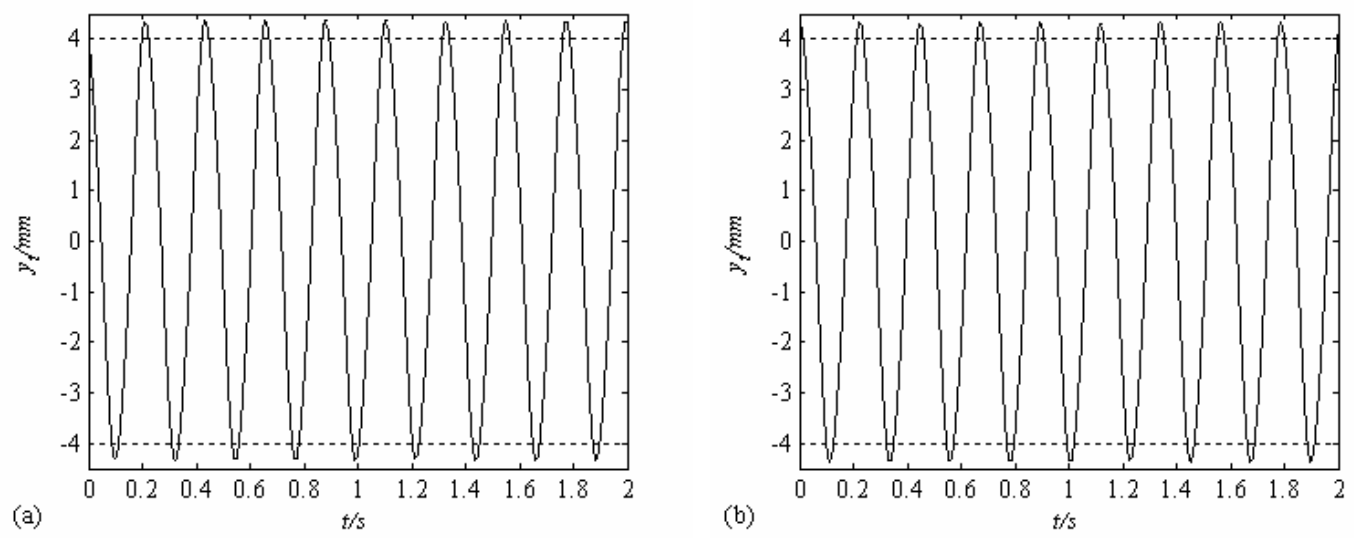

Fig. 14 Time series of the bogie frame at (a) $V=83.46 \mathrm{~m} / \mathrm{s}$ and (b) $V=83.47 \mathrm{~m} / \mathrm{s}$ showing the asymmetric chaotic attractor

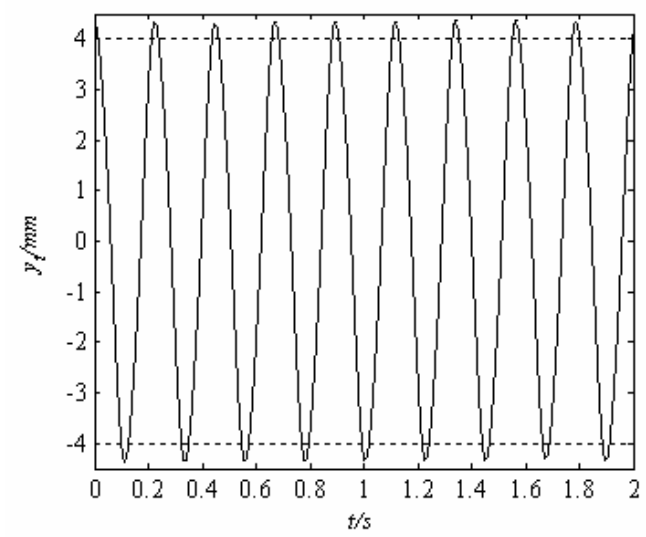

Fig. 15 A time series of the bogie frame at $V=83.48 \mathrm{~m} / \mathrm{s}$ showing the symmetric chaotic attractor

\section{Conclusions}

In the paper, the symmetric/asymmetric bifurcation behaviour and the chaotic motion of a theoretical model of a railway bogie are investigated in detail by a combination of bifurcation diagrams using the increasing and decreasing speed and reflection, and methods based on the stability and bifurcation theory of nonlinear dynamical systems.

The dynamical model of the motion of the bogie contains a complex nonlinear wheel-rail contact relation. The Shen-Hedrick-Elkins creep theory [32] describes the nonlinear interactive forces between the wheels and the rails. The equations of motion of a two-axle railway bogie system moving on a tangent and perfect track are derived 
under certain assumptions that reduce the number of degrees of freedom of the complete system. We find that the bifurcation from the stationary attractor is a sub-critical Hopf bifurcation leading to an unstable periodic solution. The unstable periodic solution gains stability at the lower 'critical speed' in a fold bifurcation. Thereby a hysteresis loop between the stationary and periodic attractors is created. For higher values of the speed, intervals are found with nonlinear dynamical behaviour such as the coexistence of two periodic motions with different amplitudes and the coexistence of a periodic motion with a symmetric or two asymmetric chaotic motions. The coexistence of the multiple steady states gives rise to hysteresis and jumps of the oscillating amplitude. This leads to a reduction of the comfort and a possible potential danger for the safe operation. Therefore the higher speeds should be avoided in the everyday operation. The asymmetric motions can be either periodic or chaotic. The development of the asymmetric motions is described. The speed intervals with the asymmetric chaotic motion are, however, small.

Chaos is often feared as a dangerous mode of operation, but it is necessary to distinguish between two types of chaos. In this work - as in the great majority of works in nonlinear dynamics - only the chaotic attractors have been investigated. In all published articles on railway vehicle dynamics the motion over the chaotic attractors looks like an erratic low amplitude disturbance superposed on a fundamental periodic hunting motion. That kind of chaos is therefore not a significantly more uncomfortable motion than the hunting motion is. In contrast transient chaos may be dangerous. Transient chaos may develop in parameter intervals where multiple attractors exist. It is characterized by an erratic motion, which may last for a long time before it settles down on an attractor, and the amplitude of the motion does not decrease monotonously like a linearly damped oscillator. The chaotic transient may exhibit large jumps of its amplitude, so large that they can be larger than the amplitudes of any of the multiple attractors the transient tends to. In the real railway world the transient chaos is under the continuous influence of the track irregularities, and it may therefore never settle at all. A case that leads to a derailment has been investigated by Pascal [40]. The chaotic transients are not only connected with the existence of multiple chaotic attractors. They 
have been observed in computer simulations of the famous low-dimensional Lorenz problem [41] in parameter intervals with either two asymmetric stationary attractors or two asymmetric periodic attractors. Since relatively little is known about the conditions for the development of chaotic attractors, the speed intervals with multiple attractors should be avoided in normal service of railway vehicles.

The modelling of the wheel-rail contact forces are one of the most important aspects in a railway vehicle model. Since the realistic wheel and rail profiles are often defined by segments of arcs of circles and polynomials, then the contact parameters are in practice often nonlinear and non-smooth functions of the lateral displacements and yaw angle of the wheel set. Due to the discontinuities in the contact parameters, the bi-contact, the multi-point contact and even the conformal contact are likely to occur in reality. On the other hand, the wheels as well as the rails are made of steel with some flexibility, so they both deform in the contact patch. This deformation has an effect on the shape and the size of the contact patch and on the direction and the values of the contact forces. Therefore, the geometry of the contacting surfaces and the nonlinear interaction between the wheels and the rails make it difficult to formulate a simple and at the same time accurate routine for the calculation of the contact forces. From this point of view, the wheel-rail contact forces used in this paper are only approximations and not very realistic ones. The more realistic models of wheel-rail contact are expected to be used in our future studies.

\section{Acknowledgment}

This research was supported by Opening Fund of State Key Laboratory of Traction Power, Southwest Jiaotong University (Grant No. TPL1106), and also supported by the National Natural Science Foundation of China (Grant No. 11072204, 11102030, 11272268) and by the Fundamental Research Funds for the Central Universities. 


\section{Appendix A}

Table 2 System parameters used for numerical analysis [42]

\begin{tabular}{ll}
\hline Value & Parameters \\
\hline$M_{t}=3000 \mathrm{~kg}$ & Mass of bogie frame \\
$I_{t x}=2260 \mathrm{~kg} \cdot \mathrm{m}^{2}$ & Roll moment of inertia of bogie frame \\
$I_{t z}=3160 \mathrm{~kg} \cdot \mathrm{m}^{2}$ & Yaw moment of inertia of bogie frame \\
$M_{w}=1400 \mathrm{~kg}$ & Mass of wheel set \\
$I_{w y}=140 \mathrm{~kg} \cdot \mathrm{m}^{2}$ & Pitch moment of inertia of wheel set \\
$I_{w z}=915 \mathrm{~kg} \cdot \mathrm{m}^{2}$ & Yaw moment of inertia of wheels et \\
$K_{p x}=1.0 \times 10^{7} \mathrm{~N} \cdot \mathrm{m}^{-1}$ & Primary longitudinal stiffness (per axle box) \\
$K_{p y}=5.0 \times 10^{6} \mathrm{~N} \cdot \mathrm{m}^{-1}$ & Primary lateral stiffness (per axle box) \\
$K_{p z}=5.5 \times 10^{5} \mathrm{~N} \cdot \mathrm{m}^{-1}$ & Primary vertical stiffness (per axle box) \\
$K_{s x}=1.5 \times 10^{5} \mathrm{~N} \cdot \mathrm{m}^{-1}$ & Secondary longitudinal stiffness (per side of bogie) \\
$K_{s y}=1.5 \times 10^{5} \mathrm{~N} \cdot \mathrm{m}^{-1}$ & Secondary lateral stiffness (per side of bogie) \\
$K_{s z}=4.0 \times 10^{5} \mathrm{~N} \cdot \mathrm{m}^{-1}$ & Secondary vertical stiffness (per side of bogie) \\
$C_{p x}=0.0 \mathrm{~N} \cdot \mathrm{s} \cdot \mathrm{m}^{-1}$ & Primary longitudinal damper (per axle box) \\
$C_{p y}=0.0 \mathrm{~N} \cdot \mathrm{s} \cdot \mathrm{m}^{-1}$ & Primary lateral damper (per axle box) \\
$C_{p z}=6.0 \times 10^{3} \mathrm{~N} \cdot \mathrm{s} \cdot \mathrm{m}^{-1}$ & Primary vertical damper (per axle box) \\
$C_{s x}=0.0 \mathrm{~N} \cdot \mathrm{s} \cdot \mathrm{m}^{-1}$ & Secondary longitudinal damper (per side of bogie) \\
$C_{s y}=6.0 \times 10^{4} \mathrm{~N} \cdot \mathrm{s} \cdot \mathrm{m}^{-1}$ & Secondary lateral damper (per side of bogie) \\
$C_{s z}=8.0 \times 10^{4} \mathrm{~N} \cdot \mathrm{s} \cdot \mathrm{m}^{-1}$ & Secondary vertical damper (per side of bogie) \\
$d_{w}=0.978 \mathrm{~m}$ & Semi spacing of primary suspension \\
$d_{s}=1.21 \mathrm{~m}$ & Semi spacing of secondary suspension \\
$l_{t}=1.2 \mathrm{~m}$ & Half of the axle distance \\
$a_{0}=0.7465 \mathrm{~m}$ & Half of track gauge \\
$r_{0}=0.4575 \mathrm{~m}$ & Centered wheel rolling radius \\
$h_{b t}=-0.081 \mathrm{~m}$ & Height of bogie frame C.G. to secondary suspension \\
$h_{t w}=0.14 \mathrm{~m}$ & Height of bogie frame C.G. to primary suspension \\
$G=8.2677 \times 10^{10} \mathrm{~Pa}$ & The resultant shear modulus \\
$W=1.1172 \times 10^{5} \mathrm{~N}$ & Axle load \\
$\mu=0.15$ & Coefficient of adhesion \\
\hline
\end{tabular}

\section{References}

[1] A.H. Wickens, Stability of high speed trains, Physics in Technology, 4 (1973) 1-17.

[2] K. Knothe, F. Böhm, History of stability of railway and road vehicles, Vehicle System Dynamics, 31 (1999) $283-323$.

[3] H. True, Dynamics of railway vehicles and rail/wheel contact, Dynamics Analysis of Vehicle Systems: Theoretical Foundations and Advanced Applications, Udine, Italy, 2007, pp. 75-128.

[4] W.-J. Chung, J.-K. Shim, Influence factors on critical speed hysteresis in railway vehicles, JSME International Journal Series C, 46 (2003) 278-288.

[5] H. True, On the critical speed of high-speed railway vehicles, Noise and Vibration on High-Speed Railways, FEUP Porto Portugal, 2008, pp. 149-166.

[6] C. Kaas-Petersen, H. True, Periodic, biperiodic and chaotic dynamical behavior of railway vehicles, Vehicle System Dynamics, 15 (1986) 208-221.

[7] C.N. Jensen, H. True, On a new route to chaos in railway dynamics, Nonlinear Dynamics, 13 (1997) 117-129.

[8] P. Isaksen, H. True, On the ultimate transition to chaos in the dynamics of Cooperrider's bogie, Chaos, Solitons \& 
Fractals, 8 (1997) 559-581.

[9] H. True, Some recent developments in nonlinear railway vehicle dynamics, Proceeding of 1st European Nonlinear Oscillations Conference, Hamburg, 1993, pp. 129-148.

[10] H. True, T.G. Hansen, H. Lundell, On the quasi-stationary curving dynamics of a railroad truck, Proceedings of the 2005 ASME/IEEE Joint Rail Conference, Pueblo, Colorado, 2005, pp. 131-138.

[11] S.P. Yang, Y.J. Shen, Bifurcations and Singularities in Systems with Hysteretic Nonlinearity, Science Press, Beijing, 2003.

[12] X.J. Gao, Y.H. Li, Q. Gao, Hunting moion and bifurcation behavior of six-axle locomotive based on continuation method, Journal of Traffic and Transportation Engineering, 9 (2009) 32-36.

[13] X.J. Gao, Y.H. Li, Q. Gao, Lateral bifurcation behavior of a four-axle railway passenger car, Transaction of the ASME, Journal of Applied Mechanics, 77 (2010) 1-8.

[14] J. Zeng, J.Y. Zhang, Z.Y. Shen, Hopf bifurcation and nonlinear oscillations in railway vehicle systems, Vehicle System Dynamics, 33 (1999) 552-565.

[15] F.J. Xia, The dynamics of the three-piece-freight truck, Informatics and Mathematical Modelling, The Technical University of Denmark, Lyngby, 2002, pp. 205.

[16] M. Hoffmann, Dynamics of European two-axle freight wagons, Informatics and Mathematical Modelling, The Technical University of Denmark, Lyngby, 2006, pp. 158.

[17] M. Hoffmann, On the dynamics of European two-axle railway freight wagons, Nonlinear Dynamics, 52 (2008) 301-311.

[18] E.D. Gialleonardo, S. Bruni, H. True, Analysis of the non linear dynamics of a 2-axle freight wagon in curves, Proceeding of 22nd IAVSD Symposium on Dynamics of Vehicles on Roads and Tracks, Manchester Metropolitan University, 2011.

[19] H. Hertz, über die Berührung zweier fester elastischer Körper (On the contact of elastic solids), Journal für die reine und angewandte Mathematik, 92 (1882) 156-171.

[20] A. Rovira, A. Roda, M.B. Marshall, H. Brunskill, R. Lewis, Experimental and numerical modeling of wheel-rail contact and wear, Wear, 271 (2011) 911-924.

[21] W. Kik, J. Piotrowski, A fast approximate method to calculate normal load at contact between wheel and rail and creep forces during rolling, Proceeding of 2th Mini-Conference on Contact Mechanics and Wear of Rail/Wheel Systems, Budapest, 1996. 
[22] J.B. Ayasse, H. Chollet, Determination of the wheel rail contact patch in semi-Hertzian conditions, Vehicle System Dynamics, 43 (2005) 161-172.

[23] X. Quost, M. Sebes, A. Eddhahak, J.-B. Ayasse, H. Chollet, P.-E. Gautier, F. Thouverez, Assessment of a semi-Hertzian method for determination of wheel-rail contact patch, Vehicle System Dynamics, 44 (2006) 789-814.

[24] L.Z. Li, J.J. Kalker, The computation of wheel-rail conformal contact, Proceedings of the 4th World Conference on computational mechanics: new trends and applications, Buenos Aires, Argentina, 1998.

[25] A. Orlova, Y. Boronenko, The anatomy of railway vehicles running gear, in: S. Iwnicki (Ed.) Handbook of Railway Vehicle Dynamics, CRC Press, Taylor \& Francis Group, Boca Raton London New York, 2006, pp. 40-83.

[26] T. Mazilu, An analysis of bogie hunting instability, U.P.B. Sc. Bull., Series D, 71 (2009) 63-78.

[27] G. Sauvage, J.-P. Pascal, Solution of the multiple wheel and rail contact dynamic problem, Vehicle System Dynamics, 19 (1990) 257-272.

[28] J. Piotrowski, H. Chollet, Wheel-rail contact models for vehicle system dynamics including multi-point contact, Vehicle System Dynamics, 43 (2005) 455-483.

[29] ArgeCare, Acradschiene: To create or approximate wheel/rail profiles, 2007, pp. 1-97.

[30] D.E. Petersen, M. Hoffmann, Dry friction and impact dynamics in railway vehicles, Informatics and mathematical Modelling, The Technical University of Denmark, Lyngby, 2003, pp. 182.

[31] D.E. Petersen, M. Hoffmann, Curving dynamics of railway vehicles, Informatics and Mathematical Modeling, The Technical University of Denmark, Lyngby, 2002.

[32] Z.Y. Shen, J.K. Hedrick, J.A. Elkins, A comparison of alternative creep force models for rail vehicle dynamic analysis, Proceeding of 8th IAVSD Symposium on Vehicle System Dynamics, Dynamics of Vehicles on Roads and Tracks, Swets and Zeitlinger, MIT, Cambridge, 1984, pp. 591-605.

[33] J.J. Kalker, A fast algorithm for the simplified theory of rolling contact, Vehicle System Dynamics, 11 (1982) 1-13.

[34] V.K. Garg, R.V. Dukkipati, Dynamics of Railway Vehicle Systems, Academic Press, New York, 1984.

[35] X.J. Gao, Y.H. Li, Q. Gao, Hunting stability and bifurcation of high-speed passenger coach, Journal of Dynamics and Control, 6 (2008) 202-207.

[36] H. True, On the theory of nonlinear dynamics and its application in vehicle system dynamics, Vehicle System Dynamics, 31 (1999) 393-421.

[37] L.F. Shampine, M.W. Reichelt, The MATLAB ODE Suite, SIAM Journal on Scientific Computing, 18 (1997) 1-22.

[38] C.N. Jensen, M. Golubitsky, H. True, Symmetry, generic bifurcations, and mode interaction in nonlinear railway 
dynamics, International Journal of Bifurcation and Chaos, 9 (1999) 1321-1331.

[39] A.H. Nayfeh, B. Balachandran, Applied Nonlinear Dynamics, Analytical, Computational, and Experimental Methods, John Wiley \& Sons, Inc., New York, 1995.

[40] J.P. Pascal, Oscillations and chaotic behaviour of unstable railway wagons over large distances Chaos, Solitons \& Fractals, 5 (1995) 1725-1753.

[41] H. True, Unpublished numerical experiments at DTU Informatics, Informatics and Mathematical Modeling, The Technical University of Denmark, Lyngby, 1999 (Personal communication).

[42] W.M. Zhai, Vehicle-track Coupling Dynamics, 2nd ed., China Railway Publishing House, Beijing, 2002. 